\title{
Hybrid Imaging of Peripheral Skeletal Disease by SPECT/ High-Resolution Flat-Panel CT
}

\author{
Christian Lohrmann ${ }^{1,2}$, Oliver Hauschild ${ }^{3}$, Philipp T. Meyer ${ }^{1}$, Michael Mix ${ }^{1}$, Peter C. Strohm ${ }^{3}$, Wolfgang A. Weber ${ }^{1,2}$, \\ and Hans-Christian Rischke ${ }^{1}$ \\ ${ }^{1}$ Department of Nuclear Medicine, University Hospital Freiburg, Freiburg, Germany; ${ }^{2}$ Molecular Imaging and Therapy Service, \\ Memorial Sloan-Kettering Cancer Center, New York, New York; and ${ }^{3}$ Department for Orthopedic and Trauma Surgery, University \\ Hospital Freiburg, Freiburg, Germany
}

The aim of this study was to determine the impact of high-resolution cone-beam CT with flat-panel detectors on the interpretation of bone SPECT in diseases of the peripheral skeleton. Methods: Forty-one consecutive patients with peripheral skeletal disease were examined with a SPECT/high-resolution CT system providing isotropic voxels of $0.33 \times 0.33 \times 0.33 \mathrm{~mm}$. High-resolution images were retrospectively analyzed by 2 readers and compared with lowresolution images obtained by filtering the high-resolution images to a lower resolution. Results: SPECT/high-resolution CT demonstrated higher diagnostic confidence scores $(1.98 \pm 0.27$ vs. 1.3 $\pm 0.45, P<0.01$ ) and better interobserver agreement ( $\kappa=0.5$ vs. $0.2)$ than SPECT/low-resolution CT. The diagnosis made by SPECT/ high-resolution CT was in agreement with the final clinical diagnosis in $95 \%$ (reader 1) and $90 \%$ (reader 2) of the cases, as compared with $59 \%$ (reader 1 ) and $60 \%$ (reader 2) of the cases for SPECT/lowresolution CT $(P<0.01)$. Conclusion: High-resolution flat-panel CT has the potential to significantly improve SPECT/CT imaging in diseases of the peripheral skeleton.

Key Words: SPECT/CT; flat-panel CT; hybrid imaging; skeletal disease

J Nucl Med 2013; 54:1323-1326

DOI: 10.2967/jnumed.112.116343

$\mathbf{R}$ adiography, CT, and MR imaging are the most frequently used imaging methods for the morphologic evaluation of the skeletal system. The most frequently performed examination to obtain functional information is bone scintigraphy with planar and 3-dimensional SPECT images (1-4). To better localize the region of increased tracer uptake and to perform attenuation correction, SPECT is now frequently combined with CT. Initial studies used a low-cost, low-resolution CT system (5-7), but more recently promising results have been published on the evaluation of bone diseases using SPECT/multidetector CT systems (8-11). However, high-resolution multidetector CT is a relatively costly technology, which significantly increases the costs of SPECT/CT systems. An

Received Oct. 31, 2012; revision accepted Feb. 4, 2013.

For correspondence or reprints contact: Christian Lohrmann, Molecular Imaging and Therapy Service, Memorial Sloan-Kettering Cancer Center, 1275 York Ave., Box 77, New York, NY 10065.

E-mail: lohrmahnl@mskcc.org

Published online May 23, 2013.

COPYRIGHT (C 2013 by the Society of Nuclear Medicine and Molecular Imaging, Inc. alternative, potentially more cost-effective, technology to obtain high-resolution images of the skeleton is cone-beam CT with flatpanel detectors (12) mounted on the gantry of the SPECT camera. The aim of the present study was to evaluate such a system in a group of patients with orthopedic diseases of the peripheral skeleton. Diagnostic confidence, interobserver agreement, and accuracy of image interpretation were compared between SPECT high-resolution CT and SPECT/low-resolution CT.

\section{MATERIALS AND METHODS}

\section{Patient Population}

A total of 41 consecutive patients ( 23 women and 18 men, ranging in age from 16 to $73 \mathrm{y}$ [mean, $51 \mathrm{y}$ ]) with a painful peripheral skeletal disease were referred by their treating orthopedic surgeons for SPECT/ CT (May 2010-December 2011) and included in this retrospective study, which was approved by the institutional review board with waiver of consent. Clinical indications were evaluation of arthroplasty and arthrodesis (14 patients), suspected osteomyelitis (13 patients), and degenerative joint disease (14 patients) (Supplemental Table 1; supplemental materials are available online at http://jnm.snmjournals.org).

Image Acquisition Protocol and Processing

${ }^{99 \mathrm{~m}}$ Tc-dicarboxypropane diphosphonate (DPD) SPECT/CT was performed using a hybrid system, BrightViewXCT (Philips), equipped with a dual-head $\gamma$-camera and a pair of low-energy, high-resolution collimators. Patients were injected intravenously with $425-760 \mathrm{MBq}$ of 99mTc-DPD (CIS Bio International). Three-phase scintigraphy was performed on 37 patients, and 2-phase on 4 patients. SPECT scans of the clinically suspected area were acquired in a $128 \times 128$ matrix with 120 projections over $360^{\circ}$ at a 20 -s acquisition time per projection. CT scans were obtained immediately after the SPECT scan using the following parameters: $80 \mathrm{mAs} / \mathrm{slice}$, peak kilovoltage of 120, 14.4-cm axial field of view in a single rotation, $17-\mathrm{cm}$ transaxial field of view, rotation speed of $12 \mathrm{~s} / 360^{\circ}$, and spatial resolution greater than 15 line pairs per centimeter at $10 \%$ modulation transfer function. SPECT images were reconstructed with an iterative 3-dimensional ordered-subset expectation maximization algorithm with built-in corrections for resolution recovery and CT-based scatter and attenuation correction (AutoSPECT Plus, Syntegra version 2.2; Philips). CT images were reconstructed using filtered backprojection with the standard parameters provided by the SPECT/CT system. The voxel size of the reconstructed images was $0.33 \times 0.33 \times 0.33 \mathrm{~mm}$. To generate images resembling low-resolution CT images used for anatomic orientation, the high-resolution flat-panel CT images were filtered with the ROVER software package (ABX $\mathrm{GmbH}$ ) to a spatial resolution of $1.32 \times 1.32 \times 10 \mathrm{~mm}$.

Radiation exposure due to administration of $99 \mathrm{~m} \mathrm{Tc}-\mathrm{DPD}$ was calculated according to ICRP publication 80 (13). To calculate the radiation 

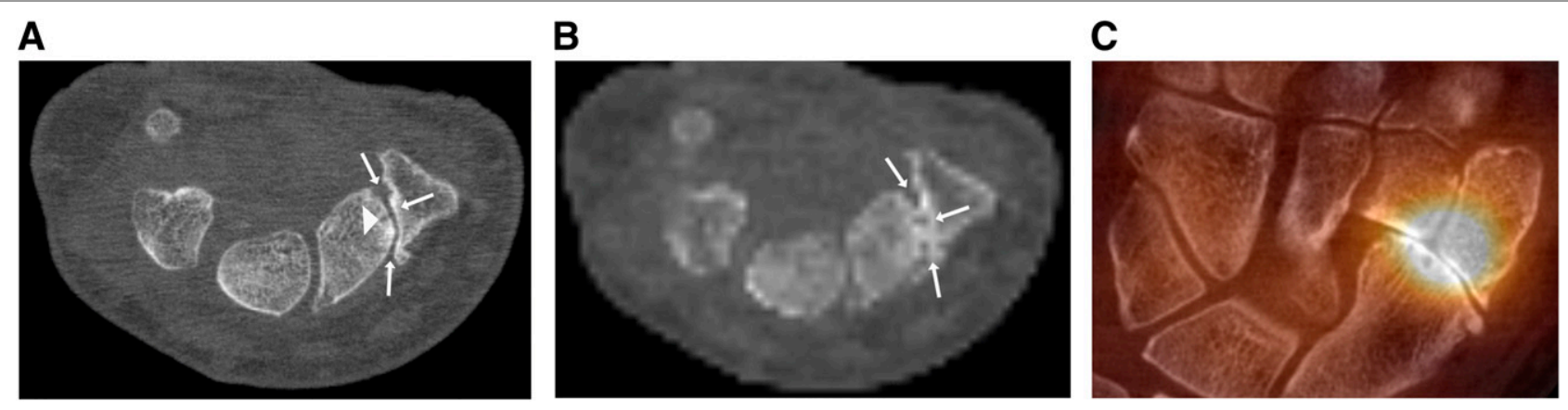

FIGURE 1. A 57-y-old woman with history of rheumatoid arthritis and pain in right hand. (A) Axially oriented high-resolution CT revealed severe postarthritic degenerative changes of scaphotrapeziotrapezoid joint, with joint space narrowing, subchondral sclerosis (white arrows), and subchondral cysts (arrowhead) (confidence score, 1); added value of high-resolution CT over low-resolution CT image is based on accurate morphologic delineation of scaphotrapeziotrapezoid joint, demonstrating severe postarthritic degenerative changes; in contrast to using low-resolution CT image, no additional examinations were needed for further evaluation). (B) Axially oriented low-resolution CT revealed osseous changes suggestive of degenerative disease of scaphotrapeziotrapezoid joint, with joint space narrowing and subchondral sclerosis (arrows) (confidence score, 2). (C) Coronal SPECT/high-resolution CT demonstrated corresponding increased tracer uptake at site of scaphotrapeziotrapezoid joint. No further pathologic tracer uptake was detected.

exposure caused by the flat-panel CT, we used a volume CT dose index of $15 \mathrm{mGy}$ as provided by the manufacturer, the axial field of view of the flat-panel detector $(14.4 \mathrm{~cm})$, and the corresponding conversion factors for the studied body region (14).

\section{Image Evaluation}

All planar and SPECT/high-resolution CT images were masked as to patient identity, ordered randomly, and reviewed on an Impax Workstation (Agfa Healthcare) using both linear gray scale and color fusion display. The low- and high-resolution SPECT/CT studies were read by 2 experienced readers in nuclear medicine and diagnostic radiology $(>5$ y experience in diagnostic radiology and $>3$ y in nuclear medicine) working independently and with all patient details masked except the referring physician's clinical question. Two reading sessions at an interval of $1 \mathrm{wk}$ were performed to minimize recall bias. In each session, the scintigraphic examinations of all 41 patients were reviewed (half of the high-resolution and half of the low-resolution images in random order). For image interpretation, a 3-point diagnostic confidence score was used. Studies received a score of 1 if a definitive diagnosis was made that explained the patient's symptoms. A score of 2 was used if the findings on the SPECT/CT study probably explained the patient's symptoms but a definitive diagnosis was not possible and follow-up or additional examinations were recommended. If SPECT/CT showed no relevant abnormalities explaining the patient's symptoms, the study received a score of 3 .

SPECT/high-resolution CT diagnoses were compared with the final clinical diagnosis. In 22 patients (54\%), intraoperative and histopathologic findings served as the reference standard. Nineteen patients (46\%) were treated conservatively, and follow-up and correlation with other imaging modalities (conventional radiography, CT, or MR imaging) were used as the reference standard. Diagnostic confidence scores and the diagnostic accuracy of SPECT/high-resolution CT and SPECT/low-resolution CT were compared by a Wilcoxon signed rank test and a McNemar test, respectively. The level of interobserver agreement was assessed by the Cohen weighted $\kappa$-coefficient.

Statistical analyses were performed with Prism 5.0 (GraphPad Software).

\section{RESULTS}

All 41 consecutive SPECT/high-resolution CT examinations were completed without technical problems, and the acquired images were of excellent diagnostic quality (Figs. 1-4). Using the SPECT/high-resolution CT images, reader 1 made a precise diagnosis (score 1) in 37 of 41 patients (90\%) and reader 2 in 30 of 41 patients $(73 \%)$. Diagnostic confidence was scored as 2 for 4 scans (10\%) and 11 scans (27\%) by readers 1 and 2, respectively. None of the SPECT/high-resolution CT images were rated with a score of 3 (Table 1; Supple-

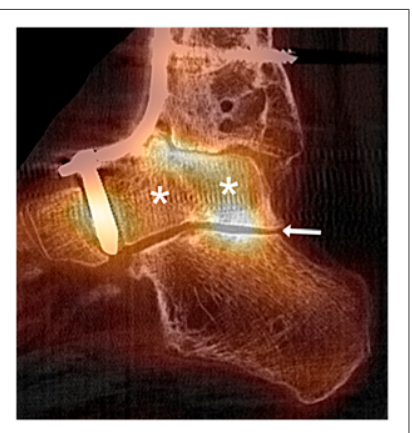

FIGURE 2. A 51-y-old woman with history of distal tibial fracture, delayed bone consolidation, and consecutive posttraumatic arthritic disease of upper ankle joint. Because of severe pain in upper ankle joint, posttraumatic arthrosis was treated with jointfusion arthrodesis. Sagittal SPECT/high-resolution CT revealed diffuse, moderately increased tracer uptake in talus bone, indicating osteomyelitis (asterisks). Elevated tracer uptake was seen in talocalcaneal joint in context of degenerative changes (arrow) (confidence score, 1 ; in this resolution CT provided morphologic substrate, and abnormalities on SPECT could be exactly localized. No further imaging was necessary). region of complex anatomy, highmental Table 1). The diagnostic confidence scores showed only modest interobserver variability $(\kappa=0.5)$. For the SPECT/low-resolution CT images, the diagnostic confidence scores were markedly lower. A definitive diagnosis was feasible in less than $20 \%$ of the patients, and most of the scans were rated with a score of 2 (Table 1; Supplemental Table 1). In addition, the diagnostic confidence scores demonstrated marked interobserver variability $(\kappa=0.2)$.

A higher mean diagnostic confidence score was obtained for SPECT/high-resolution CT than for SPECT/ low-resolution CT (1.98 \pm 0.52 vs. $1.10 \pm 0.30$ [reader $1], 1.98 \pm 0.27$ vs. $1.3 \pm 0.45$ [reader 2]). This difference was statistically significant ( $P<0.01$ for both readers).

Reader 1 made a correct diagnosis with SPECT/highresolution CT and SPECT/ low-resolution CT in 24 patients $(59 \%)$. In 15 patients (37\%), the diagnosis made with SPECT/high-resolution 


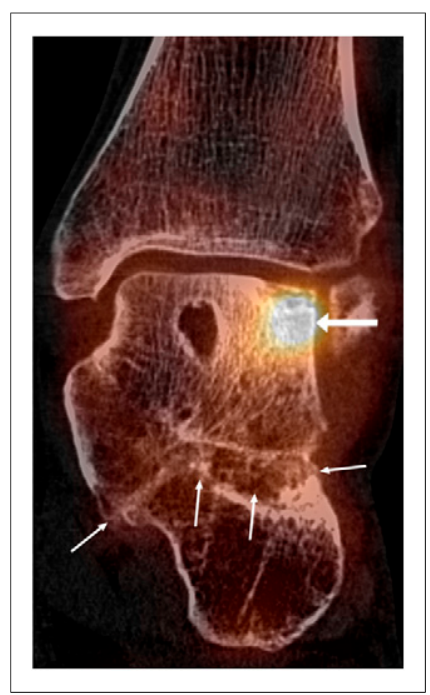

FIGURE 3. A 40-y-old woman with pain in right upper ankle joint after former talocalcaneal joint fusion. Coronal SPECT/high-resolution CT demonstrated corresponding increased tracer uptake at site of osteochondritis dissecans (thick arrow). No increased tracer uptake was seen at talocalcaneal joint, confirming complete fusion (thin arrows) (confidence score, 1; in this region of complex anatomy, highresolution CT provided exact morphologic substrate for SPECT findings, and no further imaging was needed). dissecans, arthritis, and loosening of arthroplasty could be made by the 2 readers (detailed findings are shown in Supplemental Table 2).

The effective dose per patient varied between 2.4 and $4.3 \mathrm{mSv}$ for ${ }^{99 \mathrm{~m} T c-D P D}$ bone scintigraphy. The effective dose from highresolution $\mathrm{CT}$ to the peripheral extremities varied between 0.04 and $0.11 \mathrm{mSv}$.

\section{DISCUSSION}

The results of this study indicate that high-resolution CT with flat-panel detectors significantly improves the diagnostic certainty of SPECT/CT studies of the extremities when compared with lowresolution $\mathrm{CT}$ for anatomic localization. High-resolution CT images of the peripheral skeleton can be acquired with an effective dose similar to the dose using the spiral CT technique, and the radiation exposure is low when compared with the radiation exposure due to ${ }^{99 \mathrm{~m}} \mathrm{Tc}$-phosphates. High-resolution cone-beam CT with flat-panel detectors is thus a promising technology to improve the diagnostic accuracy of SPECT studies of the peripheral skeleton and may facilitate the diagnostic workup of patients with abnormalities on bone SPECT.

Anatomic localization of abnormalities on SPECT with lowresolution CT increases specificity if focal tracer uptake is present in typical areas for degenerative changes, such as the intervertebral joints (5-7). In other cases, there is still a need for further correlation with other imaging modalities to reach a defini-

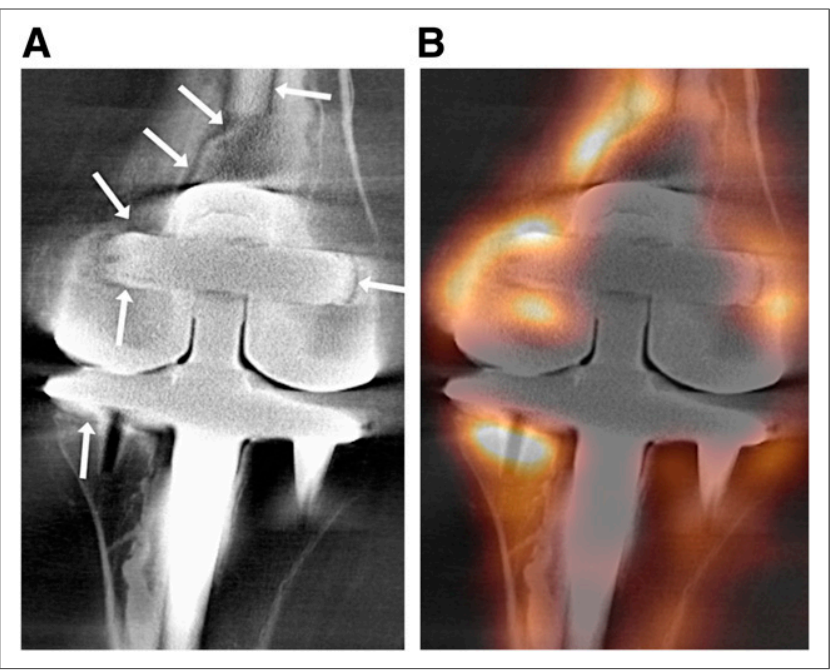

FIGURE 4. A 65-y-old woman with right knee prosthesis due to valgus deformity of right leg and consecutive severe degenerative changes of knee joint. (A) Coronal reconstruction of high-resolution CT image was able, despite scatter artifacts from prosthesis, to clearly demarcate linear lucent areas of tibia and femur adjacent to prosthesis, indicating loosening (arrows). (B) Coronal SPECT/ high-resolution CT demonstrated areas of increased tibial and femoral tracer uptake proximate to knee prosthesis, corresponding to pathologic lucent zones on CT and confirming loosening (confidence score, 1; high-resolution CT provided exact morphologic substrate for abnormalities found on SPECT, and no further imaging was needed preoperatively).

tive diagnosis, creating an additional burden for the patient and increasing costs. Flat-panel CT is a relatively recent development and has the main advantages of a high spatial resolution with isotropic voxels, volumetric coverage, and reduced metallic and beamhardening artifacts (12). The isometric voxel size allows high-resolution multiplanar images that are particularly helpful for visualizing the structure of the peripheral skeleton.

With flat-panel CT and its multiplanar reconstructions of different angles of view, localization of regions of increased tracer uptake is improved, particularly in complex anatomy such as the foot and hand. Consequently, it is possible to determine the exact bone in which uptake is increased and whether any adjacent joints are involved. Besides improving the localization of regions of increased uptake, high-resolution CT provides a detailed morphologic visualization of the skeletal structures and their diseases. Both of these factors contributed to the encouraging results in our series of patients. In about $30 \%$ of patients, a correct definitive diagnosis was made only by SPECT/high-resolution CT. Thus, future studies of

TABLE 1

Diagnostic Confidence Scores of Readers 1 and 2

\begin{tabular}{crc}
\hline \multicolumn{1}{c}{ Score } & Reader 1 & Reader 2 \\
\hline SPECT/high-resolution CT & $37(90 \%)$ & $30(73 \%)$ \\
Score 1 & $4(10 \%)$ & $11(27 \%)$ \\
Score 2 & $0(0 \%)$ & $0(0 \%)$ \\
Score 3 & $6(15 \%)$ & $2(5 \%)$ \\
SPECT/low-resolution CT & $30(73 \%)$ & $38(93 \%)$ \\
Score 1 & $5(12 \%)$ & $1(2 \%)$ \\
Score 2 & & \\
Score 3 &
\end{tabular}


TABLE 2

Diagnostic Accuracy of SPECT/Low- and High-Resolution CT

\begin{tabular}{ccc}
\hline & \multicolumn{2}{c}{$\begin{array}{c}\text { SPECT/high- } \\
\text { resolution CT }\end{array}$} \\
\cline { 2 - 3 } SPECT/low-resolution CT & Correct & Incorrect \\
\hline Reader 1 & & \\
Correct & 24 & 0 \\
Incorrect & 15 & 2 \\
Reader 2 & & \\
Correct & 25 & 0 \\
Incorrect & 12 & 4 \\
\hline
\end{tabular}

SPECT/high-resolution CT are warranted in the areas of degenerative joint disease (including osteochondritis dissecans), osteomyelitis, and arthroplasty or arthrodesis.

In patients with degenerative joint disease, high-resolution CT provides detailed information on the degree of osteophytosis and of subchondral sclerosis as well as the presence of superimposed arthritic bony erosions (Figs. 1A, 1C, and 2). In osteochondral lesions, the most important pathologic aspects are the extent of involvement of the subchondral bone plate, osteonecrosis of the subchondral bone, and separation of the osteochondral fragment. All of these can be well visualized by SPECT/high-resolution CT (Fig. 3). In patients with suspected osteomyelitis, morphologic alterations such as osteolysis, sclerosis, sequestra, or intraosseous fistula formations can be delineated by high-resolution CT, which facilitates the correct diagnosis of osteomyelitis and its complications. The advantages of high-resolution CT in patients with arthrodeses are the high spatial resolution and the reduced metallic and beam-hardening artifacts. These advantages make it feasible to image minute bridging trabeculation across the area of arthrodesis near metallic implants (Fig. 3). The evaluation of a painful arthroplasty remains a diagnostic challenge, because of the wide range of differential diagnoses. Using high-resolution CT, detailed morphologic information can be obtained and correlated with the scintigraphic findingsfor example, exact localization of linear lucent areas adjacent to arthroplasties indicating loosening (Figs. 4A and 4B).

Although high-resolution CT provided excellent images of the skeleton in the present study, the following limitations should be noted. We performed a retrospective analysis of SPECT/CT studies that were acquired as part of the clinical management of patients with extremity bone pain. CT was performed only if the interpreting physician could not establish a definitive diagnosis from the nuclear images. Therefore, one cannot conclude from our data that high-resolution CT should be performed on all patients undergoing bone scintigraphy for extremity bone pain. However, if CT is performed in combination with bone SPECT, our data indicate that high-resolution CT is considerably more effective in clarifying the diagnosis than low-resolution CT.

In this initial evaluation of SPECT/high-resolution CT, we studied a relatively heterogeneous patient population in order to define a spectrum of disorders for which high-resolution CT may be helpful. Although our findings are encouraging for the use of high-resolution CT in a variety of disorders of the peripheral skeleton, future studies are required to establish the added value of high-resolution $\mathrm{CT}$ in each of these disorders.

We investigated only the lower and upper extremities with highresolution CT. The limited current of the x-ray tube (maximum,
$80 \mathrm{mAs}$ ) causes images of the chest and abdomen to show considerably more noise and less anatomic detail. Because of the lower dynamic range of CsI-based flat-panel detectors, the contrast resolution of the studied flat-panel CT is approximately 5 Hounsfield units, which is inferior to that of multidetector CT. This inferior resolution has little impact on visualization of high-contrast objects such as bone but limits soft-tissue contrast.

\section{CONCLUSION}

This preliminary study presented promising results for hybrid imaging of peripheral skeletal disease using a SPECT/highresolution flat-panel CT system. Combining SPECT with high-resolution CT appears markedly superior to using SPECT/ low-resolution CT solely for attenuation correction and approximate localization of regions of increased tracer uptake.

\section{DISCLOSURE}

The costs of publication of this article were defrayed in part by the payment of page charges. Therefore, and solely to indicate this fact, this article is hereby marked "advertisement" in accordance with 18 USC section 1734 . No potential conflict of interest relevant to this article was reported.

\section{ACKNOWLEDGMENT}

We thank Marlies Duerr for her excellent support in examining the patients for this study.

\section{REFERENCES}

1. Hain SF, O'Doherty MJ, Smith MA. Functional imaging and the orthopaedic surgeon. J Bone Joint Surg Br. 2002;84:315-321.

2. Groshar D, Gorenberg M, Ben-Haim S, Jerusalmi J, Liberson A. Lower extremity scintigraphy: the foot and ankle. Semin Nucl Med. 1998;28:62-77.

3. Buckland-Wright JC, Macfarlane DG, Fogelman I, Emery P, Lynch JA. Technetium $99 \mathrm{~m}$ methylene diphosphonate bone scanning in osteoarthritic hands. Eur J Nucl Med. 1991;18:12-16.

4. Bellah RD, Summerville DA, Treves ST, Micheli LJ. Low back pain in adolescent athletes: detection of stress injury to the pars interarticularis with SPECT. Radiology. 1991;180:509-512.

5. Mohan HK, Gnanasegaran G, Vijayanathan S, Fogelman I. SPECT/CT in imaging foot and ankle pathology: the demise of other coregistration techniques. Semin Nucl Med. 2010;40:41-51.

6. Even-Sapir E, Flusser G, Lerman H, Lievshitz G, Metser U. SPECT/multislice low-dose CT: a clinically relevant constituent in the imaging algorithm of nononcologic patients referred for bone scintigraphy. J Nucl Med. 2007;48:319-324.

7. Scharf S. SPECT/CT imaging in general orthopedic practice. Semin Nucl Med. 2009;39:293-307.

8. Linke R, Kuwert T, Uder M, Forst R, Wuest W. Skeletal SPECT/CT of the peripheral extremities. AJR. 2010;194:329-335.

9. Gnanasegaran G, Barwick T, Adamson K, Mohan H, Sharp D, Fogelman I. Multislice SPECT/CT in benign and malignant bone disease: when the ordinary turns into the extraordinary. Semin Nucl Med. 2009;39:431-442.

10. Hirschmann MT, Iranpour F, Konala P, et al. A novel standardized algorithm for evaluating patients with painful total knee arthroplasty using combined single photon emission tomography and conventional computerized tomography. Knee Surg Sports Traumatol Arthrosc. 2010;18:939-944.

11. Pagenstert GI, Barg A, Leumann AG, et al. SPECT-CT imaging in degenerative joint disease of the foot and ankle. J Bone Joint Surg Br. 2009;91:1191-1196.

12. Reichardt B, Sarwar A, Bartling SH, et al. Musculoskeletal applications of flatpanel volume CT. Skeletal Radiol. 2008;37:1069-1076.

13. ICRP. Radiation Dose to Patients from Radiopharmaceuticals: Publication 80. New York, NY: International Commission on Radiological Protection. 1998;28:7-83.

14. Stamm G, Nagel HD. CT-expo: a novel program for dose evaluation in CT. Rofo. 2002;174:1570-1576. 